\title{
The Association Between Caustic Ingestion and Psychiatric Comorbidity Based on 396 Adults Within 20 Years
}

This article was published in the following Dove Press journal: Risk Management and Healthcare Policy

Yu-Jhou Chen, (ID) 1,2,* Chen-June Seak, (1D ${ }^{2-4, *}$ Chien-Cheng Chen, (iD) ${ }^{2,5,6}$ Tsung-Hsing Chen, ${ }^{1,2,5}$ Shih-Ching Kang, $\mathbb{D}^{7}$ Chip-jin $\mathrm{Ng}$, (1D ${ }^{2,4}$ Chao-Wei Lee, (1D ${ }^{8}$ MingYao Su, ${ }^{2,9,10} \mathrm{Hsin}-\mathrm{Chih}$ Huang, (ID) ${ }^{1,2,9}$ Chun-Hsiang

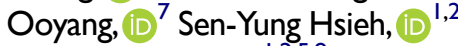
Hao-Tsai Cheng (1D) 1,2,5,9

'Department of Gastroenterology and Hepatology, Linkou Medical Center, Chang Gung Memorial Hospital, Taoyuan 33305,

Taiwan; ${ }^{2}$ College of Medicine, Chang Gung

University, Taoyuan 33302, Taiwan;

${ }^{3}$ Department of Emergency Medicine, New

Taipei Municipal Tucheng Hospital, New Taipei

City 23652, Taiwan; ${ }^{4}$ Department of Emergency

Medicine, Linkou Medical Center, Chang Gung

Memorial Hospital, Taoyuan 33305, Taiwan;

${ }^{5}$ College of Medicine, Graduate Institute of

Clinical Medicine, Chang Gung University,

Taoyuan 33302, Taiwan; ${ }^{6}$ Department of Medical Image and Intervention, Linkou Medical

Center, Chang Gung Memorial Hospital,

Taoyuan 33305, Taiwan; ${ }^{7}$ Division of Trauma

and Emergent Surgery, Department of Surgery,

Linkou Medical Center, Chang Gung Memorial

Hospital, Taoyuan 33305, Taiwan; ${ }^{8}$ Division of

General Surgery, Department of Surgery,

Linkou Medical Center, Chang Gung Memorial

Hospital, Taoyuan 33305, Taiwan; ' Department

of Gastroenterology and Hepatology,

Department of Internal Medicine, New Taipei Municipal Tucheng Hospital, New Taipei City

23652, Taiwan; ${ }^{10}$ Board of Directors and

Supervisors, Taiwan Association for the Study of Small Intestinal Disease (TASSID), Taoyuan

33305, Taiwan

*These authors contributed equally to this work

Correspondence: Hao-Tsai Cheng Department of Gastroenterology and Hepatology, New Taipei Municipal TuCheng

Hospital, No 6, Sec. 2, Jincheng Road,

Tucheng Dist., New Taipei City 236, Taiwan

(Republic Of China)

Tel +886-2-22630588 ext. 6185

Fax +886-2-22732688

Email hautai@adm.cgmh.org.tw
Purpose: High prevalence of psychiatric comorbidities (PCs) has been widely documented in caustic substance ingestion cases. However, their effect on the clinical features and prognostic outcomes remains unclear due to the paucity of discussion. We report on detailed clinical courses with long-term multifaceted outcomes and review the association between caustic ingestion and each specific PC.

Patients and Methods: The retrospective chart review included 396 adults (median follow-up, 16.6 months) with and 377 without (control group) PCs treated between 1999 and 2018 at Chang Gung Memorial Hospital. All PCs were diagnosed/confirmed by psychiatrists through face-to-face interviews.

Results: The PCs predicted serious esophagogastroduodenoscopy grading, higher rates of admission/surgery/intensive care unit stay, increments of systemic/gastrointestinal complications, and poorer 5-year overall survival rates. The poor survival among patients with PCs was highly consistent with their baseline characteristics. Significantly advanced age, more non-PCs, alcoholism, illicit drug abuse, and baseline unhealthy status resulted in statistically higher risks of severe complications and limited recovery.

Conclusion: PCs changed clinical patterns and had critical roles in the survival outcomes of caustic injury victims. Clinical awareness achieves benefit by limiting injuries in mild cases or allowing emergent interventions in severe cases. Future studies based on worldwide populations are essential for realizing geographic differences.

Keywords: caustic, corrosive, psychiatric, endoscopy, complications, long-term survival

\section{Introduction}

Caustic substance ingestion is uncommon but frequently life-threatening. The broad-spectrum complications place a heavy burden on healthcare organizations. $^{1-9}$ The extent of damage depends on the chemical properties, amount, concentration, and physical form of the ingested substances. ${ }^{1-3,5,6,10-14}$ Patient age, sex, comorbidities, complications, treatment choices, and socioeconomic status have important roles in survival outcomes and postinjury quality of life. ${ }^{1,2,4,6,7,12,13,15-21}$

Preventive measures have been widely advocated. ${ }^{1,6,10,22-25}$ However, the effectiveness of precautions appears unsatisfactory in adults, among whom suicidal intents and deliberate self-harm accounts for the majority of cases. $^{1,4,5,10,17,25}$ The associated factors include socioeconomic problems, marital/interpersonal conflicts, low educational status, and negligence in handling 
caustic substances. ${ }^{14,16,23-29}$ The high prevalence of psychiatric comorbidities (PCs) has been widely reported among caustic injury victims. $4,10,11,16-19,25,27,28,30,31$ Nevertheless, the effect of PCs on the clinical features and prognostic outcomes remains unclear because of limited information and scarce reports on long-term prognoses. To investigate the crucial aspects of caustic GI tract injury, we reviewed 396 cases within 20 years at a north Taiwan medical center.

Taiwan has an extremely high density of population and medical centers. Combined with Taiwan's National Health Insurance, the referral of caustic ingestion cases to medical centers is common, with neither traffic problems nor institutional impediments. Hence, the 20-year experience at our center included diverse scenarios. To our knowledge, this is the first research on caustic injury to simultaneously report detailed information throughout the clinical courses and review the association between longterm multifaceted outcomes and specific psychiatric diagnoses.

\section{Patients and Methods}

\section{Study Population}

This retrospective chart review of caustic ingestion included 839 adults treated between January 1999 and December 2018 at Chang Gung Memorial Hospital (CGMH) (Figure 1). We excluded 35 cases because of unclear medical records and 31 because of unclear psychiatric status. The remaining 773 cases included 396 with and 377 without (control group for comparison) PCs. All patients were adults (age $\geq 18$ ). The follow-up period of survivors ended on September 1, 2019. This study was reviewed and approved by the CGMH Institutional Review Board (IRB number: 202000583B3).

Baseline laboratory data were collected. The ingested caustic substances were confirmed by the label on containers. Strong acids and alkalis were defined by $\mathrm{pH}<2$ and $>12$, respectively. Urgent esophagogastroduodenoscopy (EGD) was performed within 24 hours after ingestion if tolerable. Those with toxic signs or other indications received plain chest radiography (CXR) or computed tomography (CT). Once a destabilized condition or respiratory difficulty was encountered, the patient was transferred to the intensive care unit (ICU) for critical care. After discharge, the patients were followed up in the outpatient clinic for at least 6 months. The intent and amount of ingestion, PCs/non-PCs, clinical treatment courses, and systemic/gastrointestinal (GI) complications were reviewed for each case. We excluded data from vague texts or equivocal narratives.

\section{Endoscopic Survey}

EGD was available around the clock at CGMH. EGD was performed by experienced endoscopists using standard Olympus upper GI endoscopes (GIF XQ-230 [9.2 mm], GIF Q-240X [9.4 mm], GIF Q-260J [9.9 mm], and GIF Q-260 [9.2 mm]; Tokyo, Japan). Patients presenting with respiratory difficulty or unclear consciousness were examined under ventilation support and general anesthesia. Caustic mucosal damage was graded using Zargar's modified endoscopic classification $(0,1,2 \mathrm{a}, 2 \mathrm{~b}, 3 \mathrm{a}$, and $3 \mathrm{~b}){ }^{32}$ Approximately half of the urgent EGDs were performed by Cheng H-T, who also reviewed the endoscopic photos of all other patients and confirmed the Zargar's grades for consistency and accuracy. The study in several patients was incomplete because of the patients' intolerance, high perforation risks, and mucosal invisibility in urgency.

\section{Psychiatric Comorbidities}

All PCs were diagnosed or confirmed by psychiatrists through face-to-face interviews, based on the Diagnostic and Statistical Manual of Mental Disorders (Fourth Edition, Text Revision [DSM-IV-TR] published in $2000^{33}$ for cases diagnosed before 2013, and Fifth Edition [DSM5] updated in $2013^{34}$ for those diagnosed after 2013). Because case enrollment spanned these two versions of diagnostic criteria, we set six categories of PCs based on their high prevalence: depressive disorders (DDs), acute stress disorder or adjustment disorder (ASD/AjD), schizophrenia spectrum disorders (SSDs), bipolar disorders (BDs), generalized anxiety disorder (GAD), and alcohol use disorder. These categories were used to reduce the influence of version differences. ${ }^{33,34}$ Cases with uncertain psychiatric diagnoses were excluded from analyses. After discharge, patients with PCs were also followed at the psychiatric clinic.

\section{Complications}

Systemic complications included aspiration injury, respiratory failure, hepatic injury (serum alanine aminotransferase or aspartate aminotransferase levels elevated to three times the normal upper limit), renal injury (serum creatinine level $>1.4 \mathrm{mg} / \mathrm{dL}$ without other noted renal diseases), and disseminated intravascular coagulation (DIC). GI complications included bleeding (melena, hematemesis, 


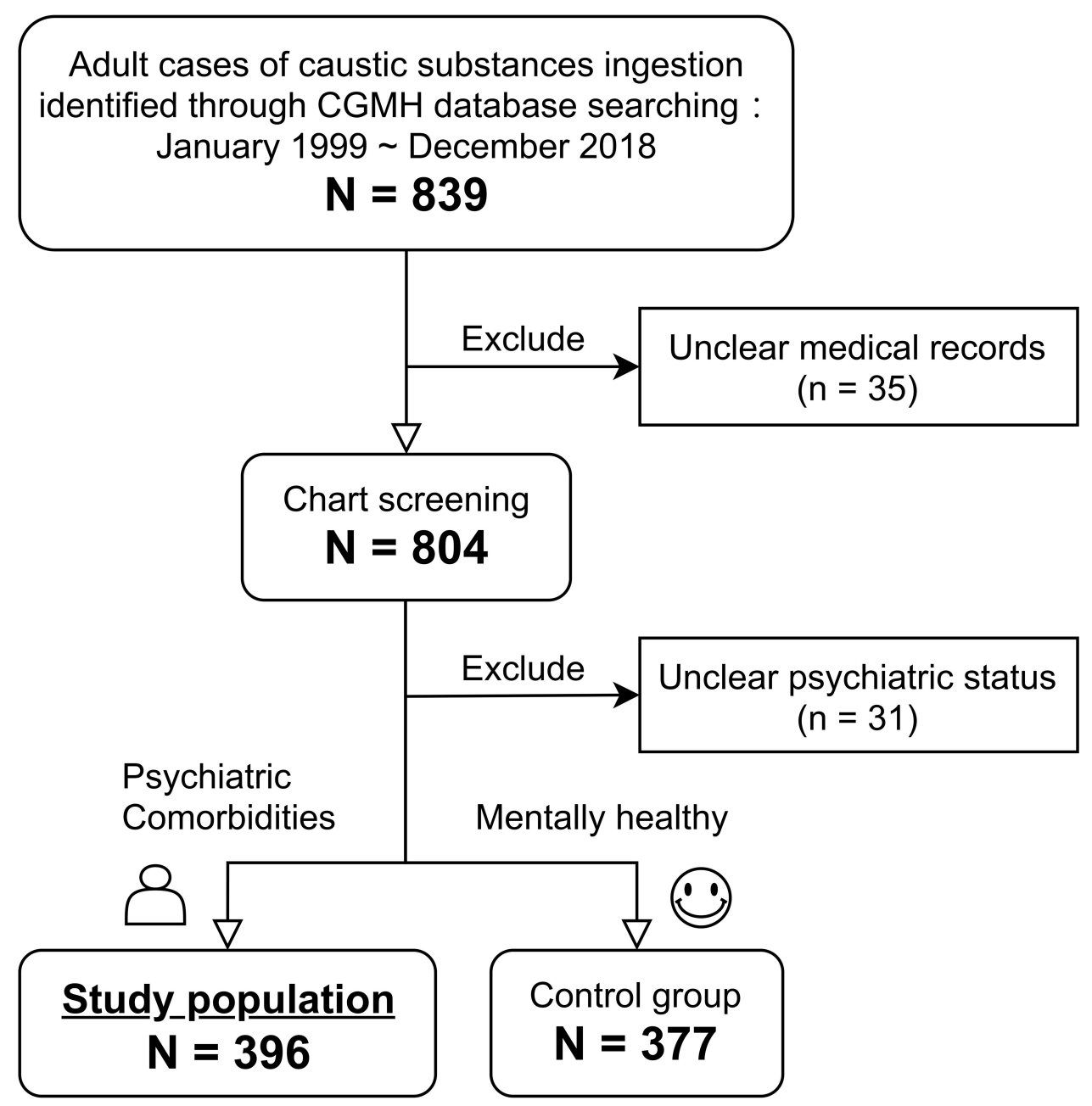

Figure I Enrollment diagram for the study population $(n=396)$ and control group $(n=377)$.

or coffee ground vomitus), perforation, fistula, and stricture formation. Perforation and/or fistula was diagnosed using CXR, CT, or endoscopy. Stricture was indicated by symptoms of dysphagia, regurgitation, or odynophagia with confirmation via endoscopy or upper GI radiography.

\section{Statistical Analyses}

Continuous variables were expressed as median with interquartile range $(\mathrm{IQR}=\mathrm{Q} 3-\mathrm{Q} 1)$ as well as whole range (minimum-maximum). Categorical variables were presented as numbers with percentages; percentage calculation excluded missing values. All statistical tests were two-sided and were performed using IBM Statistical Product and Service Solutions (SPSS), version 22 (IBM, Armonk, New York, USA). We performed independent $t$-tests or Mann-Whitney $U$-tests for continuous variables and Pearson $\chi^{2}$ tests or Fisher's exact tests for categorical variables. The univariate survival analyses were performed using the Kaplan-Meier method with Log rank tests. The Cox's proportional hazards model was performed alternatively in the multivariate setting to obtain the hazard ratio (HR) with a 95\% confidence interval (CI). A $p$ value $<0.05$ was considered statistically significant.

\section{Results}

\section{Patient Characteristics}

Among the 396 adults with PCs (158 males, 39.9\%; 238 females, $60.1 \%$ ), the median age during caustic ingestion was 46 years (IQR 29, range 18-94). Suicidal ingestion (SI) was the predominant (93.7\%) intent. There were 58 patients $(14.7 \%)$ with a history of suicide attempt by miscellaneous means; 29 had repeated caustic SI. After a survey in the emergency department, 99 patients $(25.2 \%)$ did not meet the admission criteria. For the 294 admitted patients, the median length of hospital stay (LoHS) was 14 days (IQR 17), with 20 (6.8\%) in-hospital deaths. 
Systemic and GI complications were observed in 131 (33.3\%) and 147 (37.1\%), respectively. Median follow-up was 16.6 months (range, 1 day to 254 months). The overall survival rates at 3 and 6 months, and 1, 3, 5, and 10 years were $90.7 \%, 88.6 \%, 86.1 \%, 80.1 \%, 74.2 \%$, and $53.2 \%$, respectively.

\section{Impacts of PCs}

The PCs changed the clinical courses of caustic injury in several ways (Tables 1 and 2). PCs tended to present in the elderly $(p=0.002)$ and in females $(p<0.001)$. SI and previous suicide attempts were more common, and the ingested caustic amount was statistically significantly different in those with PCs compared to those without PCs. According to the chart review, patients with PCs had significantly higher incidences of underlying hypertension ( $p=0.001)$, diabetes mellitus $(p=0.046)$, hepatitis $\mathrm{B}$ or $\mathrm{C}(p=0.042)$, and thyroid disease $(p=0.003)$.

The presence of PCs predicted more requirements for endotracheal intubation for urgent EGD $(p=0.014)$ and more frequent incomplete EGD studies $(p=0.019)$. Additionally, more systemic complications $(p=0.006)$, upper GI complications ( $p=0.006$ ), and surgical interventions $(p=0.003)$ were recorded among them. Regarding delayed sequela, PCs increased the stricture risk and thus a greater requirement for endoscopic dilatation and surgeries for esophageal strictures or gastric outlet obstructions. The 5 -year $(p=0.045)$ and 10 -year $(p=0.005)$ overall survival rates were significantly different between those with and without PCs.

\section{Characteristics of Specific PCs}

Among the 396 patients with PCs, DDs ( $n=219,55.3 \%)$ was the most common, followed by ASD/AjD ( $n=88$, $22.2 \%)$, SSDs $(\mathrm{n}=51,12.9 \%)$, BDs $(\mathrm{n}=29,7.3 \%)$, and GAD ( $\mathrm{n}=19,4.8 \%)$. Of note, SI, previous suicide attempts, and significantly larger ingestion volumes were associated with DDs, ASD/AjD, SSDs, and BDs. Patients with DDs faced the highest multifaceted risks (Appendix 1).

\section{PCs and Serious Zargar's Grades}

Patients with PCs were found with more serious caustic GI tract injuries under EGD (Table 1). Although the proportions of Zargar's grade $\geq 2 b$ were not significantly

Table I Characteristics of Caustic Ingestion Patients

\begin{tabular}{|c|c|c|c|}
\hline \multirow[t]{2}{*}{ Variables } & \multicolumn{2}{|c|}{ Psychiatric Comorbidity } & \multirow[t]{2}{*}{$p$ value } \\
\hline & Present $(n=396)$ & Absent $(n=377)$ & \\
\hline \multicolumn{4}{|l|}{ Baseline status } \\
\hline Old (>65 years old) & $98(24.7)$ & $59(15.6)$ & $0.002^{* *}$ \\
\hline Female & $238(60.1)$ & $153(40.6)$ & $<0.001 * * *$ \\
\hline Suicidal ingestions & $370(93.7)$ & $205(54.8)$ & $<0.001 * * *$ \\
\hline Previous suicide attempts & $58(14.7)$ & $6(1.6)$ & $<0.001 * * *$ \\
\hline Alcohol use disorder & $84(21.2)$ & $56(14.9)$ & $0.022 *$ \\
\hline Illicit drug abuse & $17(4.3)$ & $3(0.8)$ & $0.002 * *$ \\
\hline \multicolumn{4}{|l|}{ Caustic ingestion } \\
\hline Caustic substances & & & 0.368 \\
\hline Acidity & $232(58.6)$ & $219(58.1)$ & \\
\hline Alkalinity & $135(34.1)$ & $122(32.4)$ & \\
\hline Neutral & $4(1.0)$ & $10(2.7)$ & \\
\hline Unknown & $25(6.3)$ & $26(6.9)$ & \\
\hline Strong caustics $(\mathrm{pH}<2$ or $>12)$ & $226(60.3)$ & 209 (59.9) & 0.917 \\
\hline Ingestion amount, $\mathrm{mL}$ & $100(200)$ & $50(70)$ & $<0.001 * * *$ \\
\hline Urgent EGD survey & $385(98.0)$ & $373(99.5)$ & 0.108 \\
\hline Grade $\geq 2 b$ & $190(58.5)$ & $179(52.0)$ & 0.095 \\
\hline Grade $\geq 3 a$ & $155(47.7)$ & $127(36.9)$ & $0.005^{* *}$ \\
\hline $\mathrm{ETT}+\mathrm{MV}$ during EGD & $47(14.5)$ & $29(8.4)$ & $0.014^{*}$ \\
\hline Incomplete study & $40(12.3)$ & $24(7.0)$ & $0.019 *$ \\
\hline
\end{tabular}

Notes: Data are presented as number (\%) or median (IQR). The calculation of percentage excluded patients with missing values from chart review. The standards for asterisks are as follows: $p<0.05^{*}, p<0.0 I^{* *}$, and $p<0.00 I^{* * *}$.

Abbreviations: EGD, esophagogastroduodenoscopy; ETT + MV, endotracheal tube with mechanical ventilation; IQR, interquartile range. 
Table 2 Impacts of Psychiatric Comorbidities on Caustic Ingestion Patients

\begin{tabular}{|c|c|c|c|}
\hline \multirow[t]{2}{*}{ Variables } & \multicolumn{2}{|c|}{ Psychiatric Comorbidity } & \multirow[t]{2}{*}{$p$ value } \\
\hline & Present $(n=396)$ & Absent $(n=377)$ & \\
\hline \multicolumn{4}{|l|}{ Clinical courses } \\
\hline Admission & $294(74.8)$ & $211(56.7)$ & $<0.001 * * *$ \\
\hline Hospital stay, days & $10(17)$ & $5(9)$ & $<0.001^{* * *}$ \\
\hline In-hospital mortality & $20(6.8)$ & $13(6.2)$ & 0.774 \\
\hline ICU admittance & $84(21.7)$ & $37(10.4)$ & $<0.001 * * *$ \\
\hline ICU stay, days & $8(8.5)$ & $6(9)$ & 0.345 \\
\hline Surgical intervention & $85(21.5)$ & $50(13.3)$ & $0.003 * *$ \\
\hline Emergent surgery & $27(6.9)$ & II (2.9) & $0.012^{*}$ \\
\hline Endoscopic dilatation & $54(13.7)$ & $34(9.0)$ & $0.043^{*}$ \\
\hline Systemic complications & $|3|(33.3)$ & $90(24.3)$ & $0.006 * *$ \\
\hline Aspiration injury & $82(20.9)$ & $51(13.8)$ & $0.010^{* *}$ \\
\hline Respiratory failure & $65(16.5)$ & $31(8.4)$ & $0.00 I^{* *}$ \\
\hline Hepatic injury & $30(7.6)$ & $20(5.4)$ & 0.214 \\
\hline Renal injury & $40(10.2)$ & $23(6.2)$ & $0.047^{*}$ \\
\hline DIC & $12(3.1)$ & $8(2.2)$ & $0.44 I$ \\
\hline GI complications & $147(37.1)$ & $105(27.9)$ & $0.006^{* *}$ \\
\hline Stricture & $92(23.2)$ & $64(17.0)$ & $0.030^{*}$ \\
\hline Bleeding & $64(16.3)$ & $53(14.3)$ & 0.453 \\
\hline Perforation & $21(5.3)$ & $10(2.7)$ & 0.065 \\
\hline \multicolumn{4}{|l|}{ Overall survival } \\
\hline 3 months & $264(90.7)$ & $213(92.6)$ & 0.442 \\
\hline 6 months & $249(88.6)$ & 194 (91.9) & 0.222 \\
\hline I year & $217(86.1)$ & I7I (90.0) & 0.216 \\
\hline 3 years & $153(80.1)$ & I $38(86.8)$ & 0.096 \\
\hline 5 years & II 8 (74.2) & II4 (83.8) & $0.045^{*}$ \\
\hline 10 years & $50(53.2)$ & $65(73.0)$ & $0.005^{* *}$ \\
\hline
\end{tabular}

Notes: Data are presented as number (\%) or median (IQR). The calculation of percentage excluded patients with missing values from chart review. The standards for asterisks are as follows: $p<0.05^{*}, p<0.0 I^{* *}$, and $p<0.00 I^{* * * *}$.

Abbreviations: $\mathrm{Gl}$, gastrointestinal; ICU, intensive care unit; IQR, interquartile range.

$(p=0.095)$ different between those with $(58.5 \%)$ and without $(52.0 \%)$ PCs, the former group was found with statistically more Zargar's grade $\geq 3 \mathrm{a} \quad(47.7 \%$ to $36.9 \%$, $p=0.005$ ) corresponding to necrotic lesions.

Figure 2 further illustrates the EGD grades in each specific PC category. Compared to patients without PCs (median, Zargar's 2b), patients with DDs $(p=0.010)$ or GAD ( $p=0.006$ ) had more serious caustic injuries (median, both Zargar's 3a).

\section{Overall Survival Outcomes}

For patients with PCs, each complication, such as respiratory failure, renal injury, DIC, bleeding, perforation, and fistula, had a predicted 5-year survival rate of $<50 \%$ (univariate analyses shown in Appendix 2). The multivariate Cox's proportional hazards model is shown in Table 3. The independently significant covariates (model 1) included intubation during EGD (HR 6.38; 95\% CI 2.99-13.60), age $\geq 65$ (HR 2.80; 95\% CI 1.33-5.91), and GI complications (HR 2.30; 95\% CI 1.05-5.02). Survival curves with the Kaplan-Meier method are illustrated in Figure 3.

As aforementioned, the caustic ingestion amount was significantly higher in patients with PCs. However, model 2 in Table 3 found significance in the amount $(p=0.003)$ but not in the incidence of PCs $(p=0.376)$, suggesting that a large ingested caustic amount is a risk factor but adds no additional risk for those with PCs.

\section{Discussion}

To our knowledge, this is the first research to review the association among caustic ingestion patterns, each specific PC, and long-term survival outcomes. The presence of PCs 


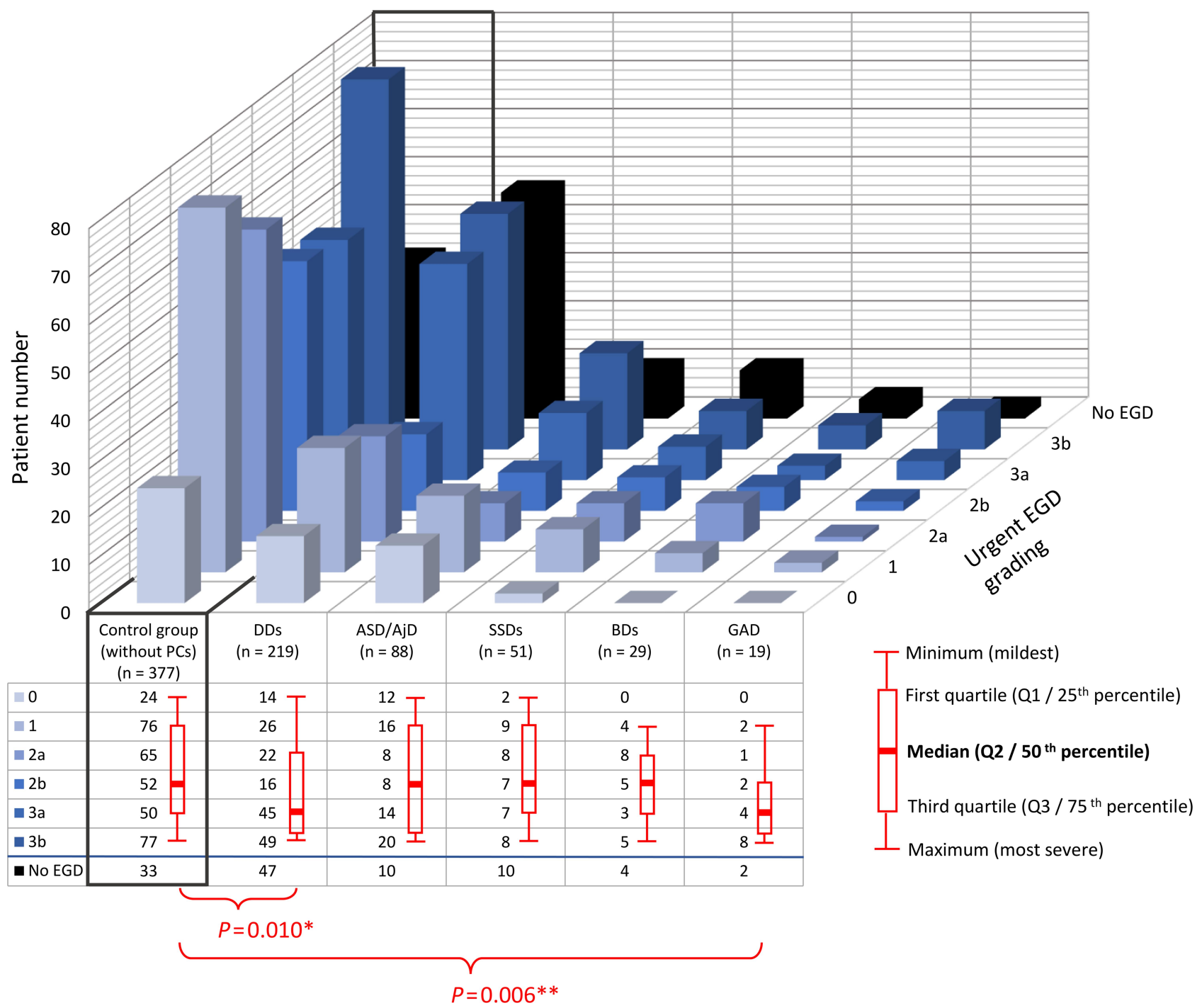

Figure 2 Urgent EGD grading for patients with each specific PC.

Notes: The standards for asterisks are as follows: $p<0.05^{*}$ and $p<0.01 * *$.

Abbreviations: ASD/AjD, acute stress disorder or adjustment disorder; BDs, bipolar disorders; DDs, depressive disorders; EGD, esophagogastroduodenoscopy; GAD, generalized anxiety disorder; PC(s), psychiatric comorbidities; SSDs, schizophrenia spectrum disorders.

predicted serious EGD grading, higher rates of admission/ surgery/ICU stay, increments of systemic/GI complications, and poor 5-year overall survival rates. Among the PCs, DDs were found to be the most common and caused the highest risk for caustic injury patients.

The incidence of caustic ingestion varies from country to country. ${ }^{1,2,5-7,10,13,14,17,19}$ Although possibly underreported, ${ }^{10,12,19}$ the annual incidence rate of caustic ingestion in Taiwan was estimated as 4.3 per 100,000 adults by the Taiwan National Health Insurance Research Database (NHIRD). ${ }^{4}$ With our 396 cases with PCs and 377 controls, our study enrolled $4 \%$ of adult cases in Taiwan. Either PCs $(49.3 \%$ vs $23.0 \%)$ or SI $(72.0 \%$ vs $38.3 \%)$ accounted for a much higher proportion in these $4 \%$ of cases than in the entire NHIRD. Similar results were also found in admission rate, LoHS, and systemic/GI complications. Collectively, our study recruited the group with relatively more serious caustic injuries.

Through analyses, PCs changed the caustic injury patterns in several ways. First, SI was predominant (93.7\%) in purpose, with the rate much higher than that in control group (5.8\%). This suggested that the link between psychiatric disorders and suicide attempts is strong regardless of caustic ingestion or other means of suicide. ${ }^{26,35-37}$

Second, advanced age was noticed among cases with PCs, in contrast to our previously reported patient group between 1999 and 2009. ${ }^{18}$ The senior population with mental illness had an increasing incidence of caustic 
Table 3 Multivariate Cox Proportional Hazards Models of Overall Survival in Patients with PCs

\begin{tabular}{|c|c|c|c|}
\hline Covariates & Case Number & HR for Death $(95 \% \mathrm{Cl})$ & $p$ value $^{a}$ \\
\hline \multicolumn{4}{|l|}{ Model I: patients with PCs $(n=325)$} \\
\hline ETT + MV during EGD & & & $<0.001 * * *$ \\
\hline No requirement & 278 & reference & \\
\hline Required & 47 & $6.38(2.99-13.60)$ & \\
\hline Age & & & $0.007 * *$ \\
\hline$<65$ & 247 & reference & \\
\hline$\geq 65$ & 78 & $2.80(1.33-5.91)$ & \\
\hline GI complications & & & $0.036^{*}$ \\
\hline Absent & 222 & reference & \\
\hline Complicated & 103 & $2.30(1.05-5.02)$ & \\
\hline \multicolumn{4}{|c|}{ Model 2: comparison with control group $(n=6 \mid 5)$} \\
\hline Ingested amount (unit: $100 \mathrm{~mL}$ ) & 615 & $1.14(1.05-1.24)$ & $0.003 * *$ \\
\hline Psychiatric comorbidities & & & 0.376 \\
\hline Absent & 316 & reference & \\
\hline Present & 299 & I.28 (0.74-2.23) & \\
\hline
\end{tabular}

Notes: ${ }^{a}$ The $p$ values were determined using Cox proportional hazards model. The standards for asterisks are as follows: $p<0.05^{*}, p<0.01 * *$, and $p<0.00 I^{* * *}$.

Abbreviations: $\mathrm{Cl}$, confidence interval; EGD, esophagogastroduodenoscopy; ETT + MV, endotracheal tube with mechanical ventilation; $\mathrm{Gl}$, gastrointestinal; HR, hazard ratio.

ingestion in the past decade. This reflects potential public health challenges for aging societies and developed countries.

Third, patients with PCs ingested significantly larger volumes of caustic agents. In our experience, the ingested volume depended on motivation and mental status. Patients with PCs more commonly underwent an "emotional surge," which led to a "swig of caustic agents." Such bolus swallowing of irritating substances dramatically increased their choking risks, which potentially explains why they had significantly more aspiration injuries $(20.9 \%, \mathrm{n}=82)$ and respiratory failures $(16.5 \%, \mathrm{n}=65)$. Tseng et $\mathrm{al}^{15}$ reported a $4.2 \%(\mathrm{n}=15)$ rate of aspiration pneumonia after intentional strong acid ingestion. They described four mechanisms: rapid ingestion of a large volume, laryngeal dysfunction among the elderly, hesitation in swallowing, and nasogastric-irrigation-induced vomiting. In our study, several patients recalled that hesitation or difficulty in swallowing limited the ingested amount. However, many more patients with PCs ingested large volumes under "emotional surge," resulting in substantially high complication risk in the respiratory system.

Cheng et $\mathrm{al}^{3}$ have emphasized the importance of early classification of caustic injuries and had implicated Zargar's $3 b$ as a valuable predictor. Our study found more advanced EGD grades in the presence of PCs. Approximately half of cases with PCs were diagnosed with necrotic GI tract injuries, equivalent to Zargar's 3a/ 3 b. Compared with mentally healthy patients, more victims with PCs either could not tolerate a complete EGD study or required ventilation support for EGD because of respiratory difficulty or unclear consciousness. They also required more additional urgent CT scans for suspected perforation or as preoperative preparation. In particular, severe caustic injury was frequently diagnosed in those with DDs and GAD (Figure 2); such patients possibly deserve higher clinical priority and more advanced examinations.

Patients with PCs had poorer long-term overall survival than mentally healthy patients (Table 2). Owing to the statistically similar chemical properties of caustic agents between the two groups, there was little connection between chemical properties and differences in survival. Furthermore, although the larger ingestion amount served as a risk factor, it had no direct impact $(p=0.376)$ on the result of the survival outcomes varying between patients with and without PCs. Univariate and multivariate analyses showed that the poor overall survival among patients with PCs was highly consistent with the GI and systemic complications. This suggests that the presence of PCs 


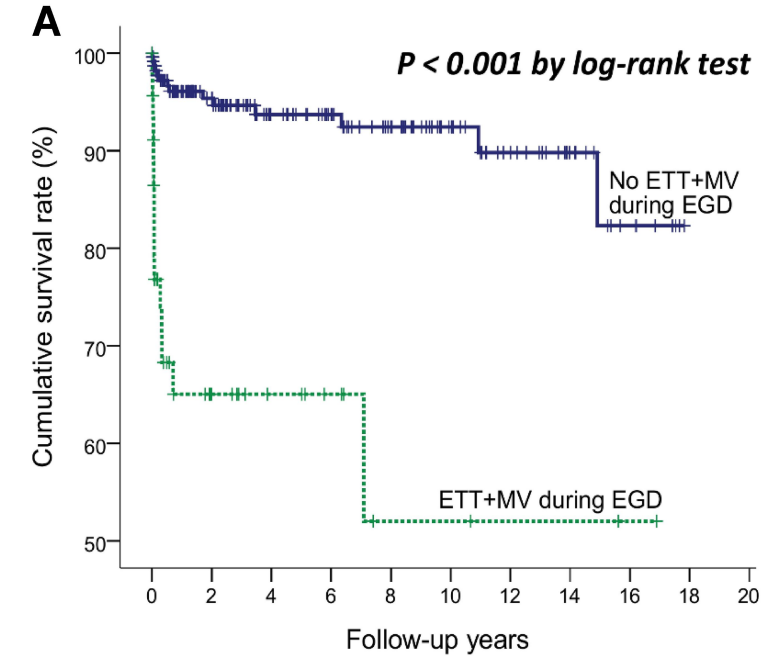

Number at risk (ETT+MV during EGD) \begin{tabular}{lccccccccccc}
\hline No ETT+MV & 277 & 130 & 91 & 77 & 60 & 41 & 27 & 16 & 8 & 0 & 0 \\
ETT+MV & 46 & 15 & 10 & 7 & 3 & 3 & 2 & 2 & 1 & 0 & 0
\end{tabular}

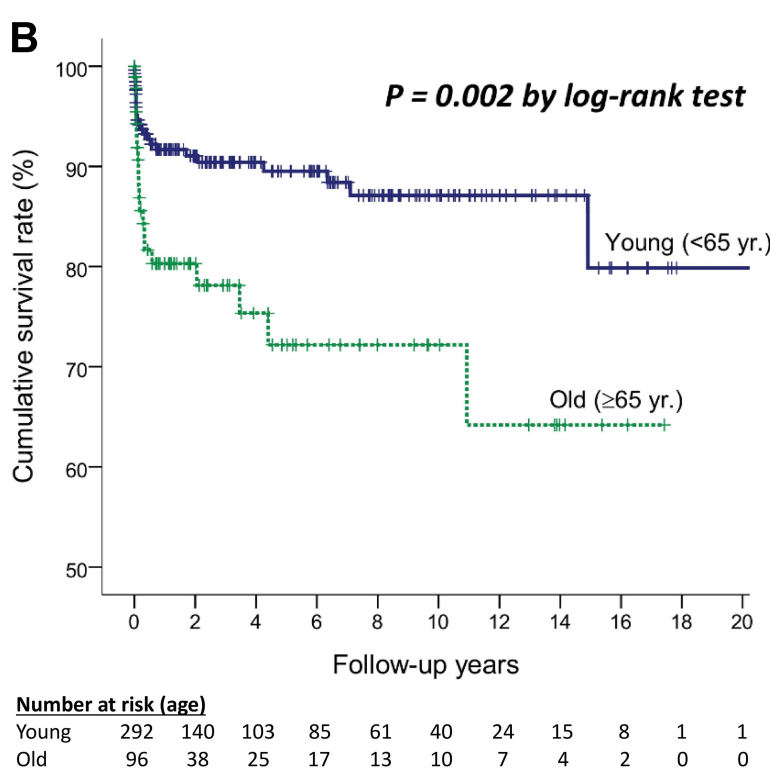

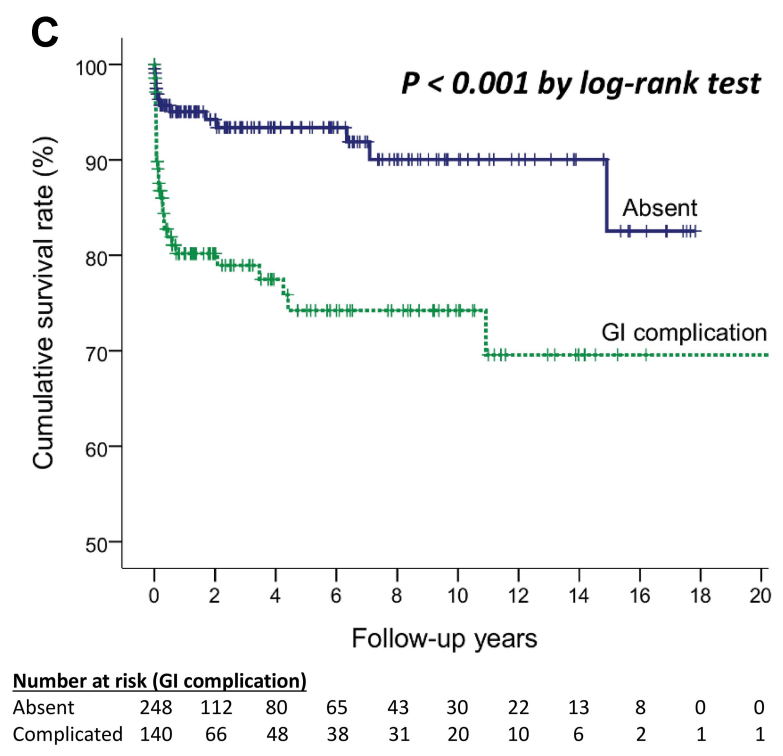

Figure 3 Overall survival analyses with the Kaplan-Meier method. (A) ETT + MV during EGD $(p<0.00 \mathrm{I})$; (B) Age ( $p=0.002)$; (C) GI complication ( $p<0.00 \mathrm{I})$. Abbreviations: EGD, esophagogastroduodenoscopy; ETT + MV, endotracheal tube with mechanical ventilation; GI, gastrointestinal.

indicates threats to caustic injury victims by "indirect means," such as significantly advanced age, more nonPCs, alcoholism, illicit drug abuse, and baseline unhealthy status, resulting in statistically higher risks of complications and limited recovery. Severe complications and delayed sequelae subsequently affected survival outcomes. Both limited recovery and longer LoHS observed in caustic ingestion cases with PCs coincided with previously reported psychological effects (particularly in DDs) on acute cardiac events. ${ }^{36}$ More healthcare resources were distributed to those with PCs regardless of whether they were within or after the acute stage of caustic injuries.
Ogunrombi et $\mathrm{al}^{16}$ have reported 27 patients with psychopathologic history, who had a limited degree of caustic injuries and better surgical outcomes. In contrast, our experiences demonstrated that PCs were predictors of severe GI tract injuries and involved greater consumption of healthcare resources, more early complications/delayed sequelae, less preservation of the native esophagus/stomach, and poorer long-term survival outcomes. Nevertheless, the population and etiology of caustic GI tract injury differ from country to country. Despite the inconsistent findings between two populations, we could integrate such valuable information and concluded that 
more attention should be paid to those with PCs. Clinical awareness brings early recognition and early management for mild cases to limit injuries, or for severe ones to receive emergent interventions.

Our study was limited by its retrospective nature and potential information bias. However, the actual caustic substances and ingested amounts might have been affected by memory lapses or clerical errors during chart recording. Additionally, the reported 20-year cases spanned two versions of psychiatric diagnostic criteria, which might have influenced our analyses. Nevertheless, the large number of cases and strict exclusion of uncertain diagnoses could compensate for the inevitable inconsistency. We studied 396 cases with detailed clinical courses and long-term multifaceted outcomes. This research, to some extent, overcame the dilemma between large sample size and detailed clinical information in the field of caustic GI tract injury. However, relatively serious scenarios accounted for a higher proportion at our center. Moreover, the etiology of caustic ingestion was roughly related to local customs, thereby presenting geographic differences. Hence, future studies based on worldwide populations are essential to comprehensively understand the association between caustic ingestion and PCs.

\section{Conclusion}

For patients with caustic substance ingestion, the presence of PCs predicted serious EGD grading, higher rates of admission/surgery/ICU stay, increments of systemic/GI complications, and poorer 5-year overall survival rates. Advanced age, more non-PCs, substance abuses, and baseline unhealthy status resulted in higher risks of complications and limited recovery from caustic injury. Severe complications and delayed sequelae affected patient survival. Clinical awareness helps limit injuries in mild cases or allows for emergent interventions in severe cases.

\section{Ethics}

Our research protocol had been approved by Chang Gung Medical Foundation Institutional Review Board (IRB number: 202000583B0; executing institution: Linkou Chang Gung Memorial Hospital, Taoyuan, Taiwan). The IRB reviewed and determined that it is expedited review according to personal information, data, documents, or specimens collected from legal biological databases without hyperlink or identifiable information that can be used for research but cannot be involved in the interests of individuals or groups. On the basis of the aforementioned statements, the IRB approves the waiver of the participants' consent in the present study.

\section{Acknowledgments}

Thanks for all the colleagues from the Department of Gastroenterology and Hepatology, Department of Psychiatry, Department of Emergency Medicine, Department of Medical Image and Intervention, Division of Trauma and Emergent Surgery, and Division of General Surgery of Linkou Medical Center, Chang Gung Memorial Hospital to help us caring the patients.

\section{Funding}

This study was supported by grants from Linkou Chang Gung Memorial Hospital Research Project: Grant CMRPG3F1931 to Dr. Hao-Tsai Cheng. The funding source had no role in the study's design, conduct, or reporting. The contents do not represent the views of Linkou Chang Gung Memorial Hospital.

\section{Disclosure}

The authors report no conflicts of interest in this work.

\section{References}

1. Hoffman R, Burns M, Gosselin S, Longo DL. Ingestion of caustic substances. $N$ Engl J Med. 2020;382(18):1739-1748. doi:10.1056/ NEJMra1810769

2. Hall AH, Jacquemin D, Henny D, Mathieu L, Josset P, Meyer B. Corrosive substances ingestion: a review. Crit Rev Toxicol. 2019;49 (8):637-669. doi:10.1080/10408444.2019.1707773

3. Cheng H-T, Cheng C-L, Lin C-H, et al. Caustic ingestion in adults: the role of endoscopic classification in predicting outcome. $B M C$ Gastroenterol. 2008;8(1):31. doi:10.1186/1471-230X-8-31

4. Chen C-M, Chung Y-C, Tsai L-H, et al. A nationwide population-based study of corrosive ingestion in Taiwan: incidence, gender differences, and mortality. Gastroent Res Pract. 2016;2016:7905425.

5. Ramasamy K, Gumaste VV. Corrosive ingestion in adults. $J$ Clin Gastroenterol. 2003;37(2):119-124. doi:10.1097/00004836-20030800000005

6. Chirica M, Bonavina L, Kelly MD, Sarfati E, Cattan P. Caustic ingestion. Lancet. 2017;389(10083):2041-2052. doi:10.1016/S01406736(16)30313-0

7. Arunachalam R, Rammohan A. Corrosive injury of the upper gastrointestinal tract: a review. Arch Clin Gastroenterol. 2016;2(2):56-62.

8. Oliva S, Romano C, De Angelis P, et al. Foreign body and caustic ingestions in children: a clinical practice guideline. Dig Liver Dis. 2020. doi:10.1016/j.dld.2020.07.016

9. Di Nardo G, Betalli P, Illiceto MT, et al. Caustic ingestion in children: 1 year experience in 3 Italian referral centers. $J$ Pediatr Gastroenterol Nutr. 2020;71(1):19-22.

10. Contini S, Scarpignato C. Caustic injury of the upper gastrointestinal tract: a comprehensive review. World J Gastroenterol. 2013;19(25):3918.

11. Struck MF, Beilicke A, Hoffmeister A, et al. Acute emergency care and airway management of caustic ingestion in adults: single center observational study. Scand J Trauma Resusc Emerg Med. 2016;24 (1):45. doi:10.1186/s13049-016-0240-5 
12. Hugh TB, Kelly MD. Corrosive ingestion and the surgeon. J Am Coll Surg. 1999;189(5):508-522. doi:10.1016/S1072-7515(99)00160-X

13. Keh SM, Onyekwelu N, McManus K, McGuigan J. Corrosive injury to upper gastrointestinal tract: still a major surgical dilemma. World J Gastroenterol. 2006;12(32):5223.

14. Elkaramany M. An overview of corrosive injury of the upper gastrointestinal tract: discussion of types, clinical evaluation, and management procedures. Adv Dig Med. 2018;5(4):115-120. doi:10.1002/ aid2.13091

15. Tseng Y-L, Wu M-H, Lin M-Y, Lai -W-W. Outcome of acid ingestion related aspiration pneumonia. Eur J Cardiothorac Surg. 2002;21 (4):638-643. doi:10.1016/S1010-7940(02)00045-3

16. Ogunrombi A, Mosaku K, Onakpoya U. The impact of psychological illness on outcome of corrosive esophageal injury. Niger J Clin Pract. 2013;16(1):49-53. doi:10.4103/1119-3077.106747

17. Christesen HB. Caustic ingestion in adults epidemiology and prevention. J Toxicol Clin Toxicol. 1994;32(5):557-568. doi:10.310 9/15563659409011060

18. Chang J-M, Liu N-J, Pai BC-J, et al. The role of age in predicting the outcome of caustic ingestion in adults: a retrospective analysis. $B M C$ Gastroenterol. 2011;11(1):72. doi:10.1186/1471-230X-11-72

19. Bonavina L, Chirica M, Skrobic O, et al. Foregut caustic injuries: results of the world society of emergency surgery consensus conference. World J Emerg Surg. 2015;10(1):44.

20. Raynaud K, Seguy D, Rogosnitzky M, Saulnier F, Pruvot FR, Zerbib P. Conservative management of severe caustic injuries during acute phase leads to superior long-term nutritional and quality of life (QoL) outcome. Langenbecks Arch Surg. 2016;401(1):81-87. doi:10.1007/s00423-015-1366-z

21. Faron M, Corte H, Poghosyan T, et al. Quality of life after caustic ingestion. Ann Surg. 2020.

22. Contini S, Swarray-Deen A, Scarpignato C. Oesophageal corrosive injuries in children: a forgotten social and health challenge in developing countries. Bull World Health Organ. 2009;87:950-954. doi:10.2471/BLT.08.058065

23. Sarioglu-Buke A, Corduk N, Atesci F, Karabul M, Koltuksuz U. A different aspect of corrosive ingestion in children: socio-demographic characteristics and effect of family functioning. Int J Pediatr Otorhinolaryngol. 2006;70(10):1791-1798. doi:10.10 16/j.ijporl.2006.06.005

24. Hutchinson G, Daisley H, Simeon D, Simmonds V, Shetty M, Lynn D. High rates of paraquat-induced suicide in southern Trinidad. Suicide Life Threat Behav. 1999;29(2):186-191.
25. Mishara BL. Prevention of deaths from intentional pesticide poisoning. Crisis. 2007;28(S1):10-20. doi:10.1027/0227-5910.28. $\mathrm{S} 1.10$

26. Petronis KR, Samuels J, Moscicki EK, Anthony JC. An epidemiologic investigation of potential risk factors for suicide attempts. Soc Psychiatry Psychiatr Epidemiol. 1990;25(4):193-199.

27. De Jong A, Macdonald R, Ein S, Forte V, Turner A. Corrosive esophagitis in children: a 30-year review. Int $J$ Pediatr Otorhinolaryngol. 2001;57(3):203-211. doi:10.1016/S0165-5876 (00)00440-7

28. Lin C, Yen T-H, Juang -Y-Y, Lin J-L, Lee S-H, Voracek M. Psychiatric comorbidity and its impact on mortality in patients who attempted suicide by paraquat poisoning during 2000-2010. PLoS One. 2014;9(11):e112160. doi:10.1371/journal.pone.0112160

29. Bertolote JM, Fleischmann A, Eddleston M, Gunnell D. Deaths from pesticide poisoning: a global response. Br J Psychiatry. 2006;189 (3):201-203. doi:10.1192/bjp.bp.105.020834

30. Havanond C. Clinical features of corrosive ingestion. J Med Assoc Thai. 2003;86(10):918-924.

31. Park KS. Evaluation and management of caustic injuries from ingestion of acid or alkaline substances. Clin Endosc. 2014;47(4):301. doi:10.5946/ce.2014.47.4.301

32. Zargar SA, Kochhar R, Mehta S, Mehta SK. The role of fiberoptic endoscopy in the management of corrosive ingestion and modified endoscopic classification of burns. Gastrointest Endosc. 1991;37 (2):165-169. doi:10.1016/S0016-5107(91)70678-0

33. American Psychiatric Association. Diagnostic and Statistical Manual of Mental Disorders. 4th ed., text revision ed. Washington, DC: American Psychiatric Pub; 2000.

34. American Psychiatric Association. Diagnostic and Statistical Manual of Mental Disorders. 5th ed. Washington, DC: American Psychiatric Pub; 2013.

35. Bachmann S. Epidemiology of suicide and the psychiatric perspective. Int $J$ Environ Res Public Health. 2018;15(7):1425. doi:10.3390/ijerph15071425

36. Brådvik L. Suicide risk and mental disorders. Int J Environ Res Public Health. 2018;15(9):2028. doi:10.3390/ijerph15092028

37. Cheng AT. Mental illness and suicide: a case-control study in East Taiwan. Arch Gen Psychiatry. 1995;52(7):594-603. doi:10.1001/ archpsyc.1995.03950190076011

38. AbuRuz ME. Pre-operative depression predicted longer hospital length of stay among patients undergoing coronary artery bypass graft surgery. Risk Manag Healthc Policy. 2019;12:75. doi:10.2147/ RMHP.S190511
Risk Management and Healthcare Policy

\section{Publish your work in this journal}

Risk Management and Healthcare Policy is an international, peerreviewed, open access journal focusing on all aspects of public health, policy, and preventative measures to promote good health and improve morbidity and mortality in the population. The journal welcomes submitted papers covering original research, basic science, clinical \& epidemiological studies, reviews and evaluations, guidelines, expert opinion and commentary, case reports and extended reports. The manuscript management system is completely online and includes a very quick and fair peer-review system, which is all easy to use. Visit http://www.dovepress.com/testimonials.php to read real quotes from published authors. 